\title{
360 Degrees Video and VR for Training and Marketing within Sports
}

\author{
By Andreas Hebbel-Seeger
}

\begin{abstract}
While the use of VR environments in the context of motor learning and training has been systematically worked on since the second half of the 1990s, especially in the area of "Serious Games," new options are emerging with 360-degree video technology and VR eyewear. For the use of these technologies, sports offer a wide range of potential usage scenarios. The close connection between 360-degree video and VR glasses and the latest consumer technology in the field of mobile devices is forcing a rapid spread, and thus also increasing the technology's importance within sports. In addition to the novelty the "immersion" aspect is currently the main argument for the use of 360-degree video and VR glasses as a projection medium. In various studies we have investigated if and how the use of 360-degree video in combination with VR-glasses has an impact on emotional, affective and physiological aspects. In this contribution I will briefly present the technology, share general usage experiences and finally discuss selected findings.
\end{abstract}

Keywords: 360 degrees video, VR, Immersion, presence, sports communication.

\section{Introduction}

Media and sports exist in a state of mutual interdependency. While media provides coverage of sports and sporting events irrespectively of time and place, sport itself fulfils all the prerequisites for acting as drivers of technological and technological development (Thieme 2014:113). With the use of radio, television and online transmissions, coverage is extended. The combination of different camera systems and perspective condenses a sports event into a new experience format. Contributing to this development is the employment of combining real life footage with artificial content in form of force-time curves, spatial tracks or promotion statistics known as "mixed reality" (Milgram et al 1994), as well as the introduction of new terminal devices (as of now this includes head mounted displays or "VR-glasses" for short) for sports media consumption.

Ultimately, the technologies mentioned above do not only open up alternative or additive possibilities of mediated sports communication, but also create a demand for new formats. "Storytelling" in sports has to be re-conceptualized whenever popular methods of steering attention are becoming limited or outdated due to users' ability to choose between several camera perspectives or let their gaze roam freely in a 360-degree projection; whenever artificial information can be accessed on demand or real projections and virtual constructions merge on different terminal devices.

\footnotetext{
* Professor for Media Management and Head of Media School at Macromedia University of Applied Sciences, Germany.

${ }^{1}$ The term "story telling" in its broadest sense describes the introduction of a message into a sequence of events. Setting and action mediate between the target audience and the message while the narrative context benefits the understanding.
} 
The development of contemporary storytelling in sports requires, in addition to sports-specific knowledge of the respective field, a basic understanding of the technological possibilities, as well as domain specific features. With this in mind I will examine the "360-degree video" in the present contribution and refer to possible projection and reception options.

\section{Feeling of Presence and Immersion}

The depiction of a sports event in the form of complete recording from the respective space of a single camera represents a fascination as well as a challenge in terms of storytelling. The aspects of presence (Singer and Witmer 1998) and immersion (Slater and Wilbur 1997) are pivotal for the communications experience. Stronger technology supports the immersion of the user into a virtual environment, and thus the closer the users believe themselves to be to the visualised events, resulting in an increasingly "real" and more intense outcome.

An important basis for this is an experience of presence that allows individuals to fully immerse into the situation and blurs the feeling of space and time into a "flow-experience" (Csikszentmihalyi 1985, Rheinberg 1995):

"Presence is defined as the subjective experience of being in one place or environment, even when one is physically situated in another. As descriped by teleoperators, presence is the sensation of being at the remote worksite rather than at the operator's control station" (Singer \& Witmer 1998: 225).

The interplay of immersion and interaction is the crucial factor in invoking an experience of presence of such kind.

In their definition of "immersion," Slater and Wilbur (1997) focus on the interface between the real environment and the virtual world communicated through media by determining the degree of immersion through aspects linked to the output devices: How many of and to what extent the human senses are reacting to stimuli generated by the device, how extensively the devices shield the user from their actual surroundings and the (technical) quality of the mediation (screen resolution, field of vision, surround sound etc.).

Despite the technical limitations of the devices discussed below, the advantages of head mounted displays (HMD) or VR glasses for 360-degree video content over screen presentations are becoming clear as the former allow for closing off from the outside world, a low-threshold access by employing everyday technology as well as native (sensitive to movement) navigation in space. Even if there is no additional "interaction" with the medium beyond the manipulation of the image section and there is "only" the possibility of grasping the events from the perspective of a camera in a fixed position in a given course of action, consumers of 360-degree videos may still have a strong experience of presence as long as the content engages the attention of the user and results in great "involvement" (Singer and Witmer 1998:227). This is also 
benefitted whenever general interest and media preparation merge into relevant content for the user.

Therefore, the experience of presence is based on "attention to continuities, connectedness and coherence of the stimulus flow" (Singer and Witmer 1998:226) and is determined by previous individual experience and interests.

The feature to give users free reign to decide over the shown image segment while wearing VR glasses, described above as a "challenge", therefore shows potential for adaption. Depending on individual interests and previously made experiences, a media based spatial adaption may happen in various ways despite employing one and the same 360-degree video and subsequently become an individualized world of experience.

Just like a good book has the ability to completely immerse its reader into a virtual world, traditional digital formats may captivate their audiences so completely that their real surroundings are upstaged, and the feeling of space and time fades into the background. Additionally, by considering the personal approach of and interest in the object of depiction, as well as a feeling of presence based on a way of media preparation as a "story", 360-degree videos show a great potential for immersion correlating with the respective means of production and device used for projection: The way of manipulating the image segment (via drag and drop on a monitor), the blocking-out of the outside world (situational context when using a screen or VR-glasses), as well as the projection's quality make an impact on the feeling of presence and thus can consequently be expected to be linked to the communication quality (Figure 1).

Figure $1.360^{\circ}$-Video, Projection Devices \& Interface

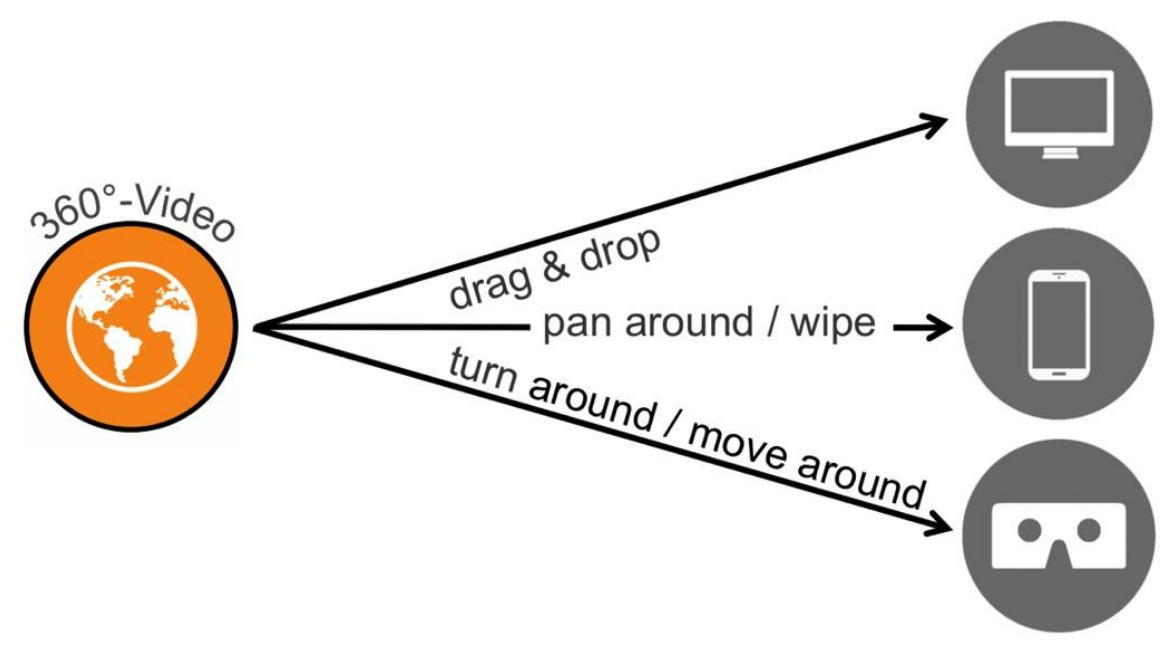

\section{0-Degree Video Technology}

360-degree cameras display the complexity of a space in complete overview. This is achieved by employing varying technological solutions: 
Single-lens solutions with a 240-degree field of vision allow for a comparatively simple video production due to all visual information being natively and directly written into a file via an image sensor. However, owing to its design the camera fails to capture an area either above or below of the recorded field of view, resulting in a literal "black spot." Depending on the communication task and on the respective documented motion activity in sporting context, this limitation is acceptable. For example, "static" views of surfboard decks, rowing boats or the sky within a rally vehicle are "thought up" by users without the immersion experience being affected.

At present, double-lens cameras are primarily being used in the consumer and the semi-professional sector. The space is imaged simultaneously in two directions via two diametrically aligned lenses, each with a field of vision of approximately 185 degrees. Both images are then merged for 360-degree projection. Depending on the device, this "stitching" takes place in real time parallel to the recording or during the follow-up with a special "stitching" software. The quality of the stitching process is determined especially at the cutting edge. The closer the objects that are picked up by both lenses are placed to the camera, the more likely artifacts are to appear in the display, meaning i.e. "fractures" in the object edges. There are substantial differences in the current generation of devices regarding this matter.

Single-lens solutions as well as high-quality double-lens cameras offer a maximum resolution of $4 \mathrm{k}$ as of yet. At first this seems to be a very high value since even cinema productions hardly work with a higher resolution. However, in the case of a 360-degree shot, apart from spherical projections in the sense of a "tiny-world-effect," ${ }^{2}$ only one image segment is viewed at all times. In case of a $4 \mathrm{k}$ image $(3,872 \times 1,936$ pixels $)$ and a typical image segment of approximately 120 degrees, a spherical projection ${ }^{3}$ results in an actual resolution of around 1,334 x 750 pixels for the observer, which is further halved when projected on a head mounted display (Figure 2).

\footnotetext{
${ }^{2}$ In this form of presentation, a spherical projection is curved over the horizontal line up to a circular representation. As a result the visualized environment seems like a sphere or a "small planet".

${ }^{3}$ In case of a spherical projection, the observer finds themselves at the centre of an imaginary sphere, on the inside of which the 360-degree image is projected. Furthermore, the observer sees only one image section which can however be moved freely both horizontally and vertically.
} 
Figure 2.Resolution and Image Sections of the Spherical Projection of a 360Degree Shot

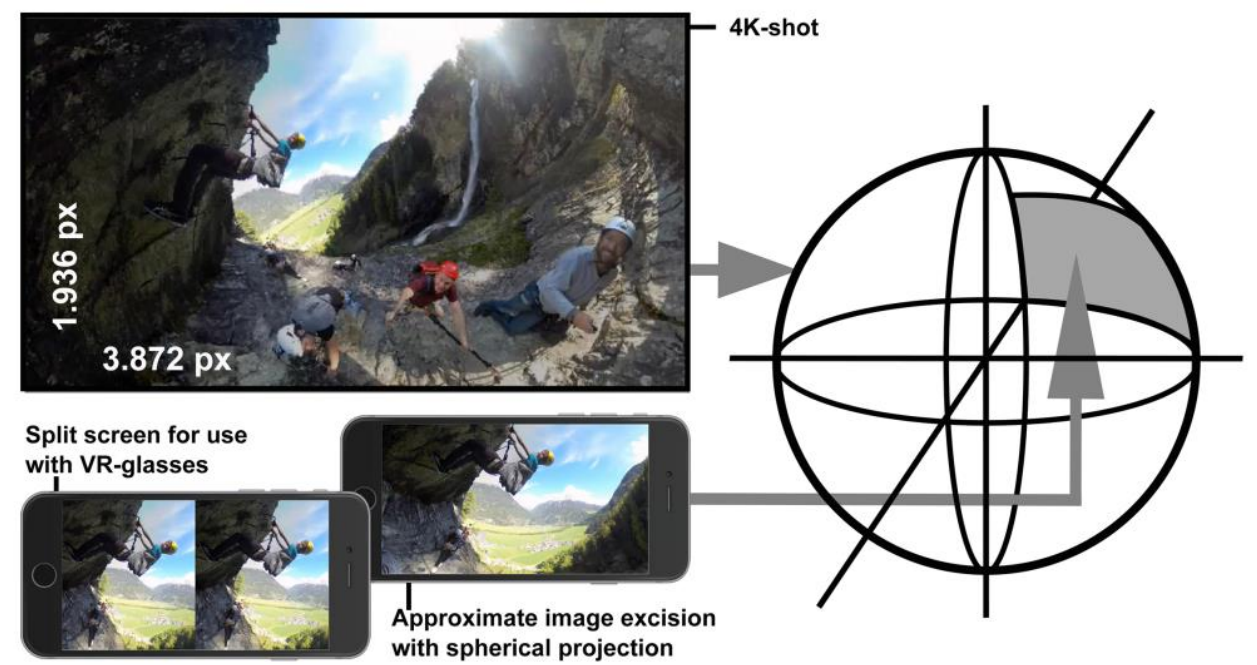

For high-quality 360-degree productions, multi-camera systems are required. These systems entail several cameras connected to each other in a special holder (a so-called "rig") in such a way that panorama, 240 degrees or even 360-degree videos can be assembled from the individual pictures taken in parallel. The total resolution in later production stages is calculated through the addition of the resolution values of the individual cameras. The angles, resolutions and frame rates of the cameras must be matched not only among each other but also to the mount. If these recordings cannot be synchronized with single image accuracy at the time of recording, this step must be repeated in post-processing either on an optical and/or acoustic basis before the assembly (the "stitching" mentioned above) can take place. The problem of overlapping image edges mentioned in connection to the double-lens camera is multiplied by the number of cameras in multi-camera systems. A further aspect is that artefacts result from missing or not precisely synchronized individual images. Even with several work steps being automated by appropriate stitching software, the effort put into post-processing remains high.

The display of 360-degree videos is basically possible on different end devices and device classes: on desktop computers or notebooks, as well as on tablet computers or smartphones. Available computing power and working memory heavily influence the display result above all. A direct reaction with smooth projection to user inputs with regard to the selection of the particular image segment requires comparatively up-to-date hardware.

In terms of software, there is a clear differentiation between server-based and client-based applications. Regarding server-based applications, the required player as well as the content are provided from an external server and delivered online and on-demand. This not only requires a sufficiently fast and stable Internet connection but also places specific requirements on the Internet browser, which are not met by all available applications. For instance, the interactive usermanipulable display of 360-degree videos via YouTube with Apple's Safari 
browser is (as of now) not possible, while the Firefox or Chrome browser on the same hardware platform allow a corresponding projection.

If the content is not available online but provided locally, software for the relevant terminal device (client) in form of a player for 360-degree content is required. The possible options for controlling the player depend on the employed device. While on desktop and laptop computers the mouse and/or keyboard are used to select the image segment ("drag and drop"), the sensitive screen surfaces of tablets or smartphones offer the possibility of control by making swiping motions with fingers ("pan around"). In addition, the software solutions for mobile devices, tablets as well as smartphones, generally also optionally respond to the position of the device in the room. The three-axis gyroscopes installed in these devices allow for the image section to be (also) manipulated via the orientation of the device. If the user wants to move the image section to the left, he or she will swivel the device accordingly. If the view is to go up, the mobile terminal is moved upwards, etc. ("move around"). This experience of potentially increased immersiveness when compared to a "classic" video projection is intensified by the use of head-mounted displays, so-called "VR glasses" that block out the outside world. In addition to proprietary complete solutions with integrated monitors responding to external computer systems (for example, Oculus Rift $@$ or HTC Vive(C), comparatively low-priced holders for smartphones, especially Google's cardboard glasses "cardboard@", have accelerated a rapid spread. When using the latter, the screen of the smartphone becomes a projection screen, which must be divided for use with VR glasses. On one hand, the exclusion of external (visual) stimuli in combination with a native choice of the image section via head movement promotes an immersive experience. On the other hand, restrictions in the image quality resulting from the comparatively close distance of the eyes to the screen and the maximum possible screen resolution of smartphones must be acquiesced. When using VR glasses, approximately half of a smartphone's available screen resolution can be efficiently used for the visualisation of the respective image segment owing to the necessary division of the screen for a separate projection for each eye. This is the reason why the display quality of 360-degree videos on VR glasses appears to be generally inferior to a full-screen projection (Figure 2).

The outlined technological limitations also encourage the occurrence of the so-called "motion sickness" (Lawson 2015). Analogous to seasickness, "see sickness" when using VR glasses also results from the supposed contradiction between the movement (acceleration) and location stimulus conceived by the equilibrium organ in the inner ear and the visual information taken in by the eyes. The occurrence and the intensity can vary significantly. Overall it can be stated, however, that the probability of occurrence correlates with the strength of camera movement in 360-degree video: The more intense and faster the camera moves and the greater the deviations from the horizontal orientation (e.g., when negotiating curves on a bicycle or the like), the more users suffer from the said effect. In self-conducted studies we further observed that activity

\footnotetext{
${ }^{4}$ The symptoms are partly synonymous with "cybersickness" or "simulator sickness" and the like (Lawson 2015:533f).
} 
during the reception of 360-degree video, such as on a bicycle gauge, levels the effects; with a still high inter individual difference. While the display quality of 360-degree video on VR glasses falls short of a full-screen projection on a computer monitor and even on a mobile terminal device due to the splitting of the screen, this very splitting promotes the depth of a spatial perception. As a general principle we are led by three different indications to a sense of spatial perception; oculomotor, stereoscopic and monocular information (Goldstein 2002).

Monocular information can be deduced from our experience by concluding a distinction between size differences, overlaps and converging lines. In this way the human being "thinks up" the depth of a room in two-dimensional projections. This cue is fundamentally the result of the observation of images and therefore is applied in a full-screen projection as well as in a divided representation.

By contrast, oculomotor information is based on convergence and accommodation. Feedback signals on the position of the eyes and shape of the lens result in interpretations of spatiality and distance. This, however, presupposes that the viewed objects, which constitute the space, are actually also at different distances from the eye. When viewing objects on a projection surface fixed at a distance from the eyes, this information source does hence not contribute.

Stereoscopic information ultimately results in the simultaneous focusing of an object with both eyes. The lateral distance of the eyes relative to one another causes a paralaxe from which the distance of the focused object is derived. The projection software of VR and 360-degree video content usually takes advantage of this functional interaction by showing the image excerpt slightly displaced for each eye; for the left eye, the image is slightly displaced to the right and vice versa for the right eye slightly to the left. As a result, a "real" stereoscopic projection is not made from a $2 \mathrm{D}$ video, since all the objects in the room have been recorded from the same position. The displacement of the image section nevertheless causes a stereoscopic effect.

\section{0-Degree Video and Augmented Reality}

"Augmented Reality" (AR) stands for an enrichment of the worldview through additional artificial information: "Augmented Reality is an immediate, interactive and realtime capable extension of the perception of one's real environment" (Broll 2013:246, author's translation). To this end, 360-degree videos can be supplemented by AR content for implementation of texts, terraristic information or biomechanical indicators. However, in the proper sense of the word, AR stands for an extension of the worldview in real time, meaning a superimposition of the real view of the environment with artifacts. In sports contexts, this can be employed in various roles, e.g. projecting the speed of movement or the jumping distance to the inner side of ski goggles (Oakley Wave (C) or the projection of the optimal swing direction and optimal ball distance on AR spectacles in golf sports, while a popular example in connection with a digital game context would be the game "Pokémon Go@". In 
the latter, the user looks at their environment on their smartphone display, which shows the respective current camera view of the device. This view is superimposed by game-relevant geodata and artificial objects such as game characters, which are integrated into the "real" environment. The possibility of use in AR contexts is generally already implemented in many VR-glasses by providing recesses for the smartphone cameras in the brackets.

Augmented Reality is a form of "Mixed Reality (MR), where real and virtual information are mixed without a precise definition of which space (virtual or real) is augmented and which space is augmenting" (Grasset et al 2011:380). Mixed Reality stands for a mixture of "real" (physical) environment and a virtual space. This mixing is basically conceivable over the entire width of a "reality-virtual continuum" (Milgram et al 1994:283),from the real to the virtual world. With extended (augmented) reality, artificial information (e.g. data superimpositions) or objects in environments with real spatial features interact with virtual objects and structures. This may occur, for example, by providing the surface of a real-world object with a virtual texture, or even the creation of virtual worlds, which are "populated" in the sense of AR by static as well as dynamic objects and subjects of the real world, i.e. by projecting threedimensional whole body scans in real time into a virtual space (figure 3 ).

Figure 3. "Reality-Virtuality-Continuum” by Milgram et al (1994:283)

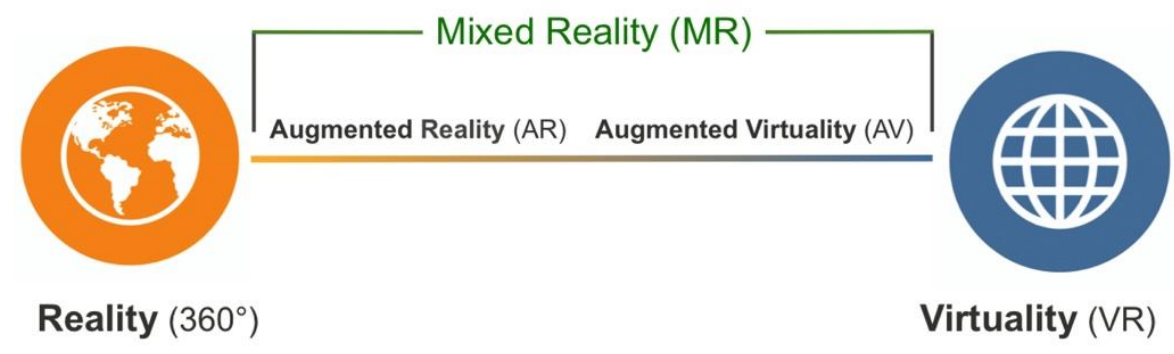

While the reality-virtuality-continuum is defined only one-dimensionally through the degree of virtualisation, current technological developments require an extension also in temporal and spatial dimensions; if, for example, the "real" world is combined across space by implementing "real" objects of a distant real world in the immediate real environment ${ }^{5}$. The temporal component becomes significant when it comes to a demarcation of 360-degree video. For as stated above, 360degrees video can be extended by artificial content. Though it does make a difference whether the "mixed reality" is generated in real-time and therefore at least potentially allows interaction with the content or whether the mixed reality is a "reality" in form of a 360-degree video, which only allows optical navigation within a fixed content-time structure.

\footnotetext{
5 "Holoportation" with Microsoft HoloLens; http://research.microsoft.com/holoportation
} 


\section{Usage of 360-Degree Video in Sports}

There is no space behind the camera in a 360-degree shot since everything is placed in the picture due to the angle. In accordance to this, the "staging" of a space in a 360-degree recording requires the entire space to be incorporated in the concept and implementation of a communication task. While classic film formats hold the attention of the viewer at a technical level by setting focal length (absolute size and zoom), choice of perspective, camera movement (pan and tracking) and mounting, these elements are largely omitted for 360-degree videos. Although it is possible to predetermine the perspective of an event between a view from either below, normal or above through positioning of the camera, due the camera's fixed focal length and the free choice of the image section in later viewing, directing attention by the definition, size and change of an image segment is not possible.

Fundamentally, it can therefore only be decided whether an event is to develop around a defined camera position, or whether the camera itself is mounted at the "point-of-action" and the dynamics of an action are directly related to the camera tracking.

If an event is to be produced primarily around the (360-degree) camera, the distance to the shooting location is crucial. For the standard focal lengths of single lens (e.g. focal length $0,88 \mathrm{~mm}$ with $360 \mathrm{Fly} 4 \mathrm{~K}$ ), double lens (e.g. Nikon KeyMission 360, focal length $1,6 \mathrm{~mm}$ each) or multi camera systems (e.g. Omni-Rig with 6 GoPro cameras; focal length 17,2mm each; wide), there are hardly any greater distances than approx. 5 to $8 \mathrm{~m}$ to individual athletes, or correspondingly somewhat larger distances to sports equipment or vehicles (for example paragliders or sailboats), since the objects otherwise appear too small. With the exception of projections on VR glasses, most 360-degree video players offer a zoom function. However, the respective resolution of the recording sets comparatively narrow limits, within which the visualisation does not yet differentiate into recognizable pixels.

Whereas hardly any proximity to the respective sporting activity can be realized with 360-degree recordings from a fixed camera position, the "proximity" to the spectator sport thrives. In the context of sporting events, a high degree of immersion can be achieved despite its mediation through media by positioning the camera in the audience area (though for the reasons mentioned above as closely as possible to the playing field). The situational character of the (almost) live experience as a spectator takes the place of the "classical" moving-imageoriented staging of a sports event through changing perspectives, different picture excerpts and a corresponding commentary. Whereas the elements outlined above in the "classical" documentation have supported the dramaturgy of a sporting event above all, the 360-degree picture appeals through its immediate "dialogue" between the sporting events on the other hand and the spectator reactions (rituals, chanting amongst fans, etc.):

"Especially interesting would be concert recordings in which the viewer would think themselves right next to the musicians on stage, or sports transmissions with a seat directly on the sidelines of the playing field. These 
fields can be expected to be "normal" first - an evolution of classical television. The view from the perspective of the Formula 1 driver by camera has been long established - now imagine that one could look around freely and selfdetermined from this perspective" (Matzat 2016, author's translation). Even if attempts to communicate sporting events in the form of 360-degree livestreams are already being implemented and the first companies specialising in this form of sports communication are particularly pushing onto the US market (nextvr.com or vokevr.com), at least currently the data transfer rates necessary for a 360degree livestream are just as much of challenge as the limitations of the terminal devices described above. For the principle of "being in the midst instead of only being present", the immersive participation in an event, presupposes the use of head-mounted displays, or simply VR glasses, which are experiencing a rapid spread with the use of mobile terminal devices as projection surfaces. In terms of the quality of the presentation VR glasses still have a significant potential for advancement and must tap into it if a monetarisable access to (sports) events is to be established.

A solution focused on the sporting activity as opposed to the spectator is installation on sports equipment or on athletes themselves. As an extension of this basic idea of so called "Action Cams", the users can later look not only through "through the eyes" of the respective athlete but slip into the person themselves- Though they would have no influence over the visualised action, they would be able to look around "just like in real life". On one hand, highly immersive experiences can be conveyed which could otherwise not be accessible to the user; e.g. driving a Formula 1 car, sailing on a racing catamaran or a boardercross race on a snowboard, etc. On the other hand, new options for selfreflection in learning and optimisation processes in sports present themselves. For the actual athletes, use of 360-degree video recordings of their own sports activity enables them not only to watch their own actions from an external view and compare it to their own inner view, as was possible in traditional recordings, but also check whether and possibly what information could have been derived from certain situations, if they had looked elsewhere; this achieved by freely choosing the image section in the aftermath of the actual activity. This appears to be equally relevant to technical and tactical decisions, f.e. in sailing or for operational decisions, e.g. as a route post in motorsport (Hebbel-Seeger \& Vohle 2016). The mediation of a new perspective on sport not only aims at a change of perspective, as the users slip into the role of the actor, but explicitly also includes the surrounding space. In this sense, a user not only perceives the view of, for example, a rower in a narrow and positionsensitive boat, but can also feel the differentiated experiences resulting from a cyclical movement in a uniform environment such as a channel or from the dynamics of a competition with other boats in front of appealing scenery.

If sports in the 360-degree space are not only to simply happen but to be staged, the particular relationship between the activity itself and the environment in which it takes place requires clarification:

If, for example, a rudder is accompanied by a 360-degree camera on a training trip on the Rotrigo de Freitas in front of the "Zuckerhut" (Olympic 
Channel 2016) during the summer Olympics in Brazil in 2016, the boat becomes the "vehicle" for a touristically influenced appropriation of space and the sporting activity thus subordinated to this goal. Even if the user can look around in all directions just like they would on an excursion steamboat or a harbour tour, the route and cutting are adjusted so this analogy actually occurs to the observer: The action is framed in terms of dramaturgy by the launching of the boat at the beginning and the landing at the end. The rudder forms a fixed point and together with the visible stern of the boat provides orientation in the space, which is also maintained through the cuts. These cuts in turn strive for the combination of scenes in which, as far as possible, impressions are conveyed in all directions and the variety of the space is compressed.

In an advertising spot presented by the Audi Sailing Team 2016, however, space has a different relationship with activity (Audi Sailing 2016). Here the space is merely the projection surface on which a highly dynamic activity is about to unfold. At first, the beach is a bridge that is not only superficially mediating between the product of the sponsor (a car) and the content of the spot, but also a metaphor for a transition and a change in perspective that takes the viewer from the familiar terrain and role of an observer into a "new" world. This world is henceforth defined by the immediate sports equipment and the athletes controlling this equipment. The width of the space outside of the boat does not provide any kind of anchor for the user, who could keep the eye, so that the attention can remain "on board". Cut scenes from out of a helicopter stand only in alleged contradiction to this. Instead they promote the desired effect, in which the 360-degree space is limited by the helicopter on one side and the sailboat on the other side. Between these two high-tech devices (helicopter and Hitech sailing skiff), the blue of the sky and of the water emerge as connecting elements, on the moving surface of which the white lines of the keel water leads to the dinghy and underpin the dynamics of the happenings.

Largely irrespective of the relationship between the environment and the movement activity in the space, and just as regardless of projection kind as either a 360-degree video or "classic" monitor projection, storytelling in sport must be based on the logic of movement and the inner dramaturgy of a competition. In the context of 360-degree videos, some of the same (e.g. with regard to an audio commentary from the off), in part less (in particular with regard to the selection, limitation and combination of camera shots and settings), and, in some cases, other possibilities (especially with regard to the customization of the appropriation of space) of presentation are available. Accordingly, neither all spaces nor every movement activity are equally suitable for the staging of a 360-degree video. First of all, one must be able to defend the decision why, with a defined communication goal, a space-encompassing all-round view should be possible. Furthermore the challenge of the next step, which would be to provide a structuring order in a complex 360-degree setting with a free and basically uncontrolled selection of the image segment by the recipients, must be addressed. This order can either be created by the arrangement of objects in the space or by the orientation towards "learned" communication patterns in which the recipients follow, for example, gazes or pointing fingers from protagonists or in the use of situational experiences, 
e.g. as a spectator of a sporting event in an arena or participants of a guided tour of a museum.

Owing to the high immersion potential of 360-degree video, especially in combination with a head-mounted display (VR glasses), this technology is highly suitable for transporting "experiences" space- and time-transcendently through media. In addition to technical aspects, which are determined by the formal quality of media production and projection, the inner design, meaning the staging of sports in the space, plays a central role in the development of this potential. On the one hand, the space must provide sufficient exploration possibilities, and thus have an internal "justification" for the media choice).On the other hand, users must not lose themselves in the exploration of the space in the sense of the implied communication goal. The challenge is therefore to guide the users. This is achieved by an orientation towards "learned" communicational behaviour via appropriate situating of the content: When my opposite points in a certain direction in a communicational situation, my gaze usually follows the gesture. As a spectator of a sports event, I can distinguish between the dramaturgy of the staged movement and viewer behaviour; suppose I have learned to look in the "right" direction at the "right" time.

Therefore, alternative indications for a guided dialogue must be sought in situational contexts, which are at first mostly unknown to users. In addition to clumsy visualizations in the form of graphical markings, directing attention via a surround sound would be a possible solution. However, such a technological solution increases the expenditure during production and so far is only supported by a few terminal devices in practical use. Further options are based on the choice and the change (cut) of the camera position as well as the inner and outer setting. This means, on one hand, the positioning of the (inner) action in the sense of storytelling and on the other hand, the staging of the (external) usage situation with respect to the touchpoint and the its available infrastructure (technical output devices and localization of the user e.g. via seating furniture that could possibly be rotated and can be pivoted). Overall, 360-degree video, especially in combination with VR glasses, is an innovative technology. This technology is currently still being met with a high curiosity motivation (Schmalt \& Langens 2009:159). With an abatement of this curiosity motivation on the user side, the demands for formal as well as content quality of the 360-degree content increase; (not only) in the sense of a successful brand communication it becomes increasingly important to work from the experimental use of an innovative technology (with its positive radiation effects on a brand resulting from the "mere" proximity to innovation) towards a recognizable and meaningful media application.

\section{Empirical Studies}

In various studies we have examined effects of the use of 360-degree video in different fields of application in sport. In the present paper, I will focus on 
two studies, one of which concentrates on the effects of media use during sports training, while the other study deals with brand communication in sport.

\section{0-Degree Video in Brand Communication}

A central aspect of brand communication is the experience. Brands should become perceptible, tangible and comprehensible for consumers. With both the goals of customer loyalty as well as transporting brand attributes and brand values are pursued, we correspondingly wondered what influence the immersion and experience of presence of 360-degree video on VR glasses possess compared to a "classic" video reception via a monitor on the communication success. A 360-degree video clip of the automotive brand Audi's sailing sport (Audi Sailing, 2016) served as the subject of investigation. We presented this clip to two test groups on different terminal devices (VR glasses: Zeiss VRone with iPhone 6 vs. screen: iMac 21.5," each with headphones) with different projection forms (Spherical Projection vs. Planar Projection) and subsequently queried for brand recognition and attributes (Figure 4).

Figure 4.Treatment Groups
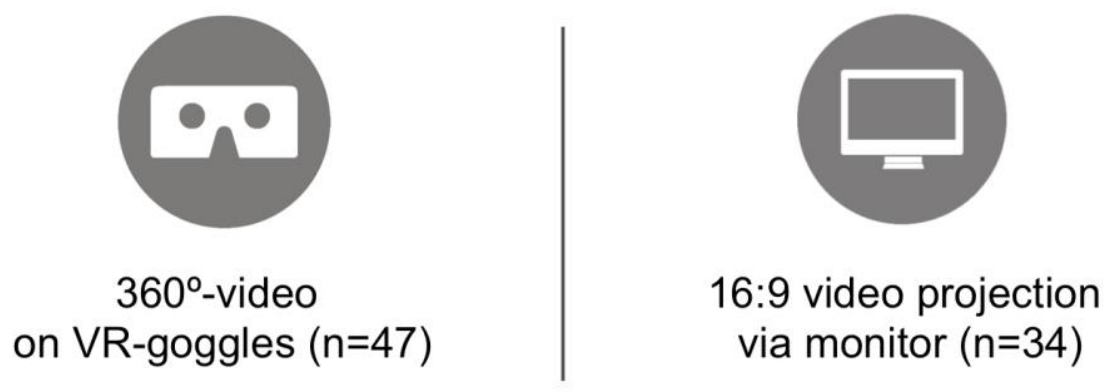

While the first group with VR glasses only saw a section of the 360-degree video in a spherical projection and was able to control it by head and body movements, the screen group received continuously and unchangeably the complete 360-degree shot in (planar) view. Subjects of this study were persons of the target group aged between 18 and 35 years with a driving license (passenger car driving license), which after the treatment (video reception) rated attributes according to their appearance in the video on a 5-stage scale between "fully applies" and "does not apply at all." The queried attributes were based on eight aspects of the "Audi brand DNA" according to Schmidt \& Vest (2010:73) and an added 12 additional items.

We have assumed in our research hypothesis that the stronger feeling of presence and immersion in the "VR treatment" compared to the "screen treatment" leads to a higher quality of experience, as a result of which the communication of brand attributes intended with the sponsorship commitment of Audi will be more successful and thus lead to stronger approval.

As a matter of fact, a comparison between the two experimental groups $(\mathrm{n}=47$ in the VR group vs. $\mathrm{n}=35$ in the screen group) reveals significant differences in attribution ( $t$-test with a significance threshold of $\mathrm{p}<0.05$ ), while no 
correlations can be detected via alternating variables (gender, preferred car brand, possession of vehicle). For one brand attribute of Audi ("confident") including seven additional items ("perfect, dynamic, passionate, individual, attractive, youthful, refined"), we queried significantly higher identification values from the screen group, whereas no group-specific differences for six other brand attributes ("sporty, innovative, modern, high-quality, stylish, successful") and one additional item ("fascinating") were to be found. Solely one additional attribute ("athletic") is significantly stronger associated by the VR group with the content consumed in the treatment compared to the screen group (Figure5).

Figure 5. Items with Significantly Higher Approval
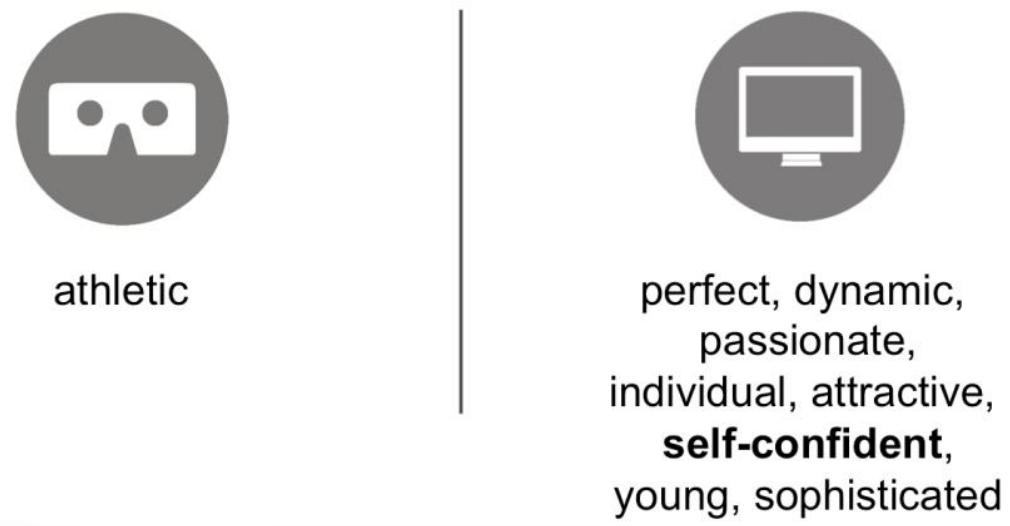

One brand attribute ("traditional") and three additional items ("boring, vintage, old") were significantly more strongly rejected by the VR group than by the screen group in relation to the content consumed in the treatment (Figure 6).

Figure 6. Items with Significantly Higher Rejection

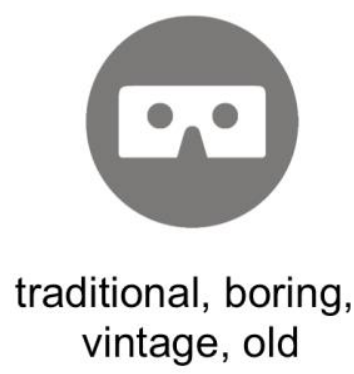

We interpret the result in the way that not every "experience" presupposes a "feeling of presence": The showing of the 360-degree space as a spherical 16:9 projection on a monitor conveys the fascination of sailing at least as well as the projection via VR glasses. Though the latter show a higher immersion potential, they also only display a portion of the overall event. To this extent it appears plausible that the "monitor" group evaluates relevant attributes of the content more strongly than the subjects of the "VR" group. However, the aspects 
based on "immediate" experience and the assumption of an "active role" in the visualised events, are assessed significantly more strongly by the "VR" group. It is thus possible to prove a differentiation where the experience of presence becomes the message, where consumers "emphasize" with the protagonists and the "athletics" of the sport become tangible.

By contrast, we hold the novelty of the consumption of 360-degree videos on VR glasses, which is (yet) largely independent of content accountable for the stronger rejection of attributes that stand out from a traditional (media) experience ("boring, old, traditional, vintage").

The results therefore support the assumption made in section 5 that the immersive potential of 360-degree video does not reveal itself to be of communicative surplus value per se, but is dependent on the message and the object of presentation.

\section{0-Degree Video in Physical Training}

Fitness devices such as bicycles, rowing ergometers or treadmills simulate real-world activity under standardized conditions. The cyclic movement lacks the aspect of locomotion in the simulation.

Therefore, we explored in the context of an exploratory study whether and under which conditions the simulation of locomotion by consumption of a 360degree video depicting the "real" activity of cycling and associated distractions caused by varying spatial impressions influence the motivation and perceived exertion of athletes on a bicycle gauge.

For this purpose we accompanied 24 subjects on four training sessions each within a period of three weeks. At the beginning of each session, the subjects had a warm-up training consisting of 10 minutes on a bicycle gauge. The subjects consisted of 10 female and 14 male visitors of a fitness studio in Hamburg, which usually use the bicycle ergometer as a warm-up. The subjects were divided into two groups with regard to their usual training frequency: "casual athletes" with maximum of two training sessions per week and "intensive athletes" with three or more training sessions per week. Both the "occasional athletes" and the "intensive athletes" were further divided into a treatment group and a control group (Figure 7).

\section{Figure 7. Sample Allocation}

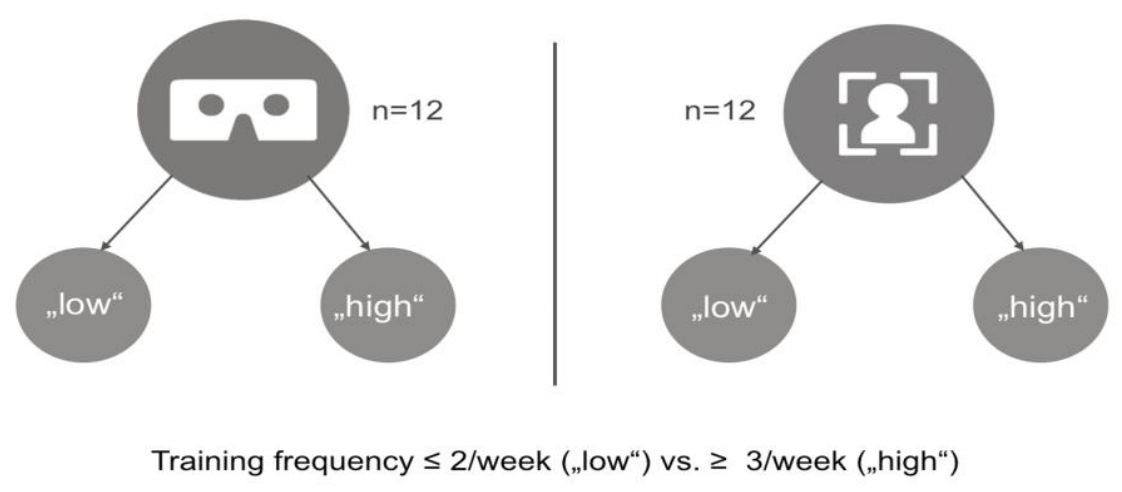


While the treatment groups were accompanied on the bicycle ergometer by a 360-degree video of a bicycle tour through the "Hamburger Stadtpark" (Hamburg City Park) from the "first person view" via a head mounted display", the control groups were not subjected to any interventions in addition to their athletic activity.

In the sense of the question at hand, we have concentrated on two aspects of investigation, the general sports motivation and the individual stress sensitivity (Figure 8).

Figure 8. Survey Aspects

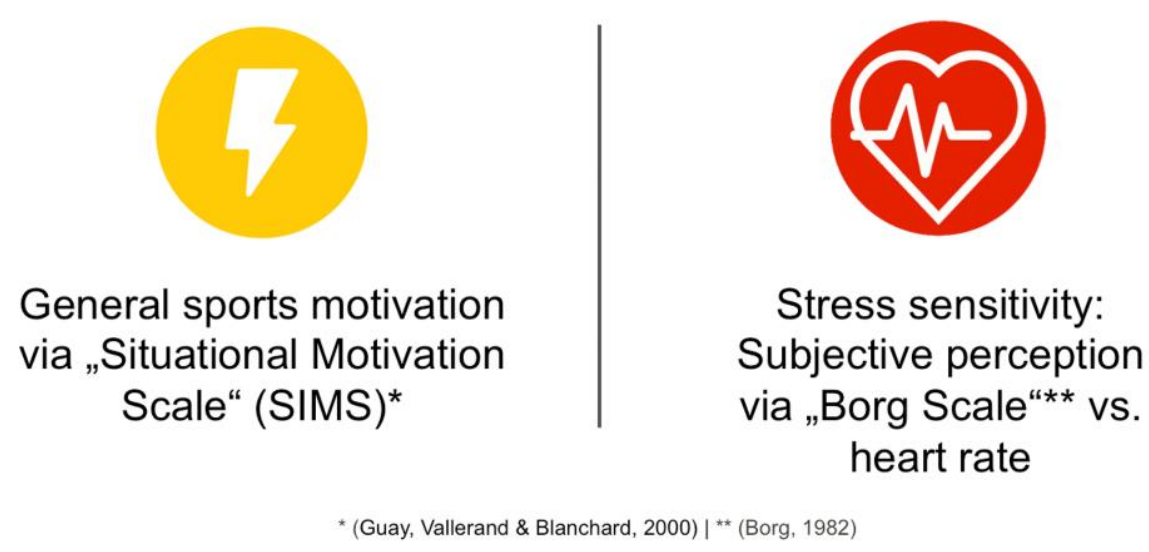

The general sports motivation of the subjects was enquired and compared to the first and last training session on the "Situational Motivation Scale" (Guay et al 2000).

The Borg scale (Borg 1982) was used to measure the individual perceived exertion. The values enquired of the subjects on this basis were then correlated with the actual measured heart rate.

With regard to the individual stress sensitivity, it was found that the "occasional athletes" with the 360-degree video treatment differed from the control group in that they were able to evaluate their training more easily subjectively than objectively (Figure 9).

\footnotetext{
${ }^{6}$ LG VR glasses "360 VR" with LG smartphone "G5"
} 
Figure 9. Subjective Stress Prediction of Probands with Low Training Intensity
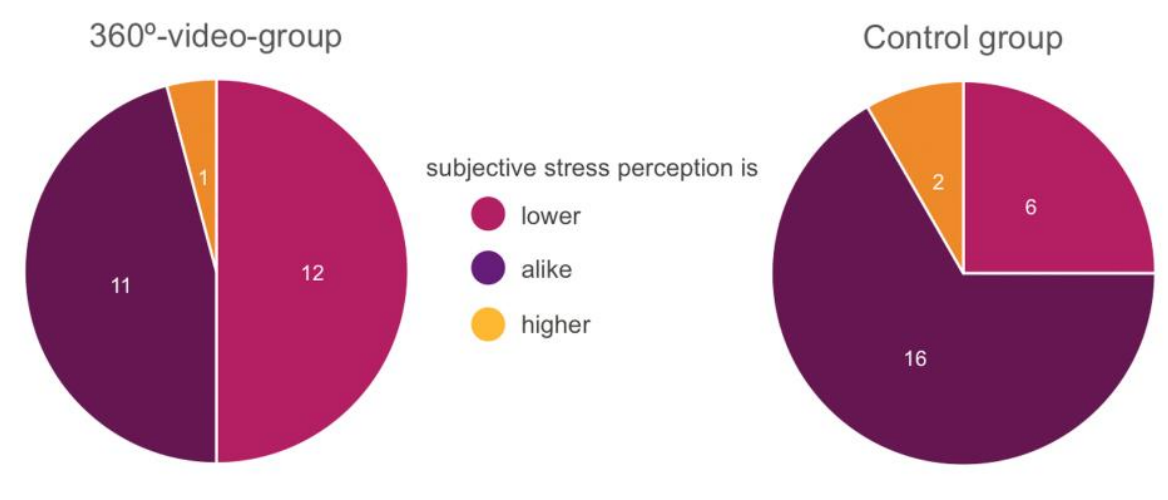

Meanwhile, the results of the "intensive athletes" were reversed. Compared to the treatment group, the control group was more likely to perceive their training as subjectively easier than determined by a measuring technique (Figure 10).

Figure 10. Subjective Stress Prediction of Probands with High Training Intensity
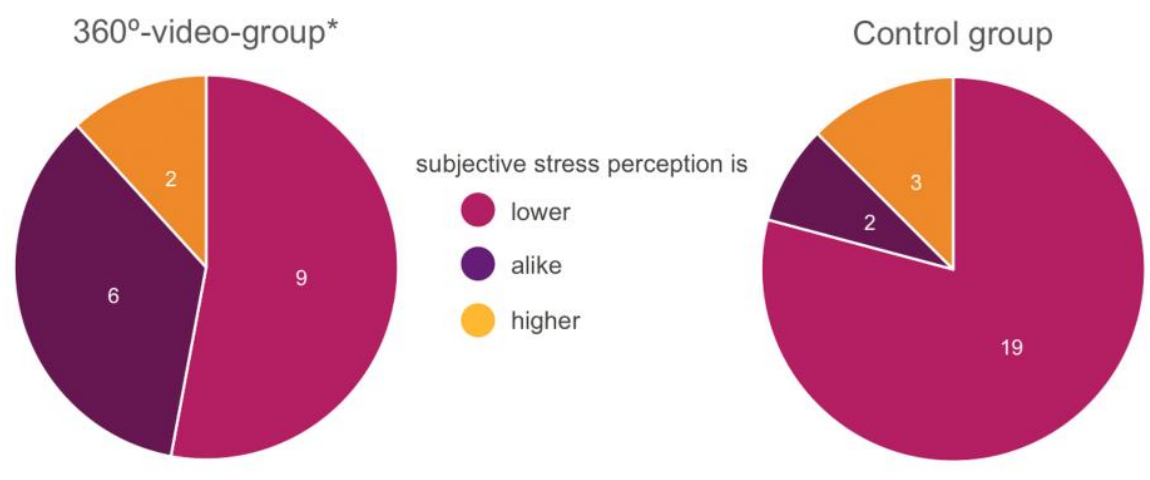

In the same way, with regard to the aspect of general sports motivation, the treatment group of the "occasional athletes" seems to benefit from the use of media, whereas no group differences can be observed in the "intensive athletes".

Overall it can be concluded that the use of 360-degree video with fitness machines seems to have positive effects on people with a low training frequency (and potentially less intrinsic motivation); an effect that was more pronounced in our study in women than in men.

In the case of persons with a higher training frequency (and higher intrinsic motivation), the use of 360-degree video leads to distractions and hampers selfperception.

This study outlined here is, as mentioned at the outset, an exploratory one with a comparatively small sample size. Nonetheless, the results suggest that the "experience of presence" through the use of 360-degree video in training processes does not necessarily generate positive effects. In the presented study, interferences between the "experience of presence in a virtual place" and selfperception in the physical world in subjects with elaborate domains-specific 
training experience (and correspondingly trained self-perception) had a negative effect on the aspect of perceived exertion.

\section{Conclusions}

Overall 360-degree videos, especially in combination with VR glasses, are an innovative technology. This technology is currently being met with a high curiosity motivation (Schmalt\&Langens 2009:159). With an abatement of the novelty stimulus on side of the users, the demands on the formal as well as the content quality of the 360-degree content increase. Not only in the sense of successful brand communication, it becomes increasingly important to evolve from the experimental use of an innovative technology to a recognizable and meaningful media application.

First exploratory studies, such as the one outlined in the present paper, have already given clear indication of the fact that 360-degree videos have a high potential for adaptation for athletic training processes by exploring the content individually at least regarding the image segment. However, individual dispositions and experiences can interfere with the experience of presence and may also produce adverse effects.

This aspect is to be investigated further in subsequent studies as well as to research other influencing variables. The use of 360-degree video in learning and optimization processes is of particular interest, for which it is necessary to examine whether and to what extent general movement experience and the development of sports-specific knowledge and skills are variables of successful media use.

\section{References}

Audi Sailing (2016) A breathtaking Audi sailing event in VR! Retrieved from https:// youtu.be/GcxOMDC8wBE. [Accessed 14 November 2017].

Borg G (1982) Psychophysical bases of perceived exertion. Medicine and Science in Sports and Exercise 14( 5): 377-381.

Broll W (2013) Augmentierte Realität. In R Dörner, W Broll, P Grimm and B Jung (eds) Virtual und Augmented Reality (VR/AR), 241-294. Berlin, Heidelberg: Springer Vieweg.

Csikszentmihalyi M (1985)Das Flow-Erlebnis. Stuttgart: Klett-Cotta.

Grasset R, Mulloni A, Billinghurst M, Schmalstieg D (2011)Navigation Techniques in Augmented and Mixed Reality: Crossing the Virtuality Continuum. In B Furht (ed) Handbook of Augmented Reality, 379-407. New York, Dordrecht, Heidelberg, London: Springer.

Goldstein EB.(2002)Wahrnehmungspsychologie. Heidelberg, Berlin: Spektrum.

Guay F, Vallerand RJ, Blanchard C (2000) On the Assessment of Situational Intrinsic and Extrinsic Motivation: The Situational Motivation Scale (SIMS). Motivation and Emotion 24(3): 175-213. 
Hebbel-Seeger A \& Vohle F (2016) Innovative Videoformate im Sport zwischen Training und Marketing. Retrieved from https://spark.adobe.com/page/tXV3K/. [Accessedat 17 January2017].

Lawson BD (2015) Motion Sickness Symptomatology and Origins. In KS Hale and KN Stanney (eds) Handbook of Virtual Environments: Design, Implementation, and Applications, 531-599. Boca Raton: CRC Press.

Matzat L (2016) Das endgültige Medium? Über das Potenzial von Virtual Reality für den Journalismus. Fachjournalist. Retrieved from http://www.fachjournalist.de/das-end gueltige-medium-ueber-das-potenzial-von-virtual-reality-fuer-den-journalismus/. [Accessed 07.03.2017].

Milgram P, Takemura H, Utsumi A, Kishino F (1994) Augmented Reality: A class of displays on the reality-virtuality continuum. SPIE, Telemanipulator and Telepresence Technologies (2351): 282-292. Retrieved from http://etclab.mie.utoronto.ca/pub lication/1994/Milgram_Takemura_SPIE1994.pdf. [Accessed 19 January 2017].

Olympic Channel (2016) $360^{\circ}$ Rowing in Rio. Retrieved from https://youtu.be/85Vdre 8MAl8. [Accessed 14 November 2017].

Rheinberg F (1995) Flow-Erleben, Freude an riskantem Sport und andere "unvernünftige" Motivationen. In J Kuhl and H Heckhausen (eds), Enzyklopädie der Psychologie, 101-118. Göttingen: Hogrefe.

Schmalt H-D, Langes TA (2009)Motivation(4. überarbeitete und erweiterte Auflage). Stuttgart: Kohlhammer.

Schmidt D \& Vest P (2010) Die Energie der Marke. Ein konsequentes und pragmatisches Markenführungskonzept. Berlin, Heidelberg: Springer.

Singer MJ, Witmer BG (1998) Measuring Presence in Virtual Environments: A Presence Questionnaire. Presence: Teleoperators and Virtual Environments 7(3): 225-240.

Slater M, Wilbur S (1997) A framework for immersive virtual environments (FIVE): Speculations on the role of presence in virtual environments. Presence: Teleoperators and Virtual Environments 6(6): 603-616.

Thieme L (Hrsg) (2014) Spezielle Aspekte des Sportmanagements. Zur Sportartikelindustrie, dem Controlling in Sportclubs und Angebotsaspekten in Sportvereinen. Berlin: epubli. 
